\title{
Adaptação Transcultural da The Stigma Scale para o Português Brasileiro: Estudo Piloto
}

\author{
Nicolas de Oliveira Cardoso ${ }^{1}$ D \\ Pontifícia Universidade Católica do Rio Grande do Sul - PUCRS, Porto Alegre-RS, Brasil \\ Breno Sanvicente-Vieira (iD \\ Pontifícia Universidade Católica do Rio de Janeiro - PUCRJ, Rio de Janeiro-RJ, Brasil \\ Wagner de Lara Machado (D), Irani Iracema de Lima Argimon(D) \\ Pontifícia Universidade Católica do Rio Grande do Sul - PUCRS, Porto Alegre-RS, Brasil
}

\section{RESUMO}

O estigma da doença mental (EDM) é apontado como um agravante dos quadros de transtorno mental, sendo recomendada pelo ministério da saúde a realização de pesquisas que auxiliem na identificação e intervenção a esse fenômeno. Este estudo teve como objetivo realizar a adaptação transcultural da The Stigma Scale, para o português brasileiro e verificar seus parâmetros psicométricos preliminares. O método deste estudo foi estruturado em cinco etapas: 1. obtenção da permissão para adaptação; 2. tradução do instrumento; 3 . síntese e avaliação por especialistas; 4. retrotradução e avaliação por especialistas; 5. estudo piloto. A versão brasileira da The Stigma Scale foi aplicada em 23 pacientes psiquiátricos. O instrumento apresentou boas evidências de validade de conteúdo $(k=0,81)$ e consistência interna $(\alpha=0,87)$. Os participantes do estudo piloto não reportaram dificuldades relacionadas à compreensão dos itens. Os autores originais da The Stigma Scale aprovaram a versão brasileira do instrumento.

Palavras-chave: tradução; validação; estigma da doença mental; saúde mental.

\section{ABSTRACT - Transcultural adaptation to Brazilian Portuguese of the Stigma Scale: a pilot study}

Mental illness stigma (MIS) has been indicated to be an aggravating factor for mental disorders, therefore, the national department of health recommends the performance of research that seeks to assist in the identification of this condition and suggest intervention strategies. This study aimed to perform the transcultural adaption to Brazilian Portuguese of the Stigma Scale and to verify its preliminary psychometric parameters. The methodology used was structured in five steps: 1. obtaining permission to adapt; 2. translation of the instrument; 3. synthesis and evaluation by specialists; 4 . back-translation and specialist evaluation; and 5. a pilot study. The Brazilian version of The Stigma Scale presented good content validity $(k=0.81)$ and internal consistency $(\alpha=0.87)$. The pilot study sample did not report difficulties related to the understanding of the items. The original authors of the Stigma Scale approved the Brazilian version of the instrument.

Keywords: translation; validation; mental illness stigma; mental health.

\section{RESUMEN - Adaptación Transcultural de la Escala de Estigma para el portugués brasileño: estudio piloto}

El estigma de la enfermedad mental es considerado un agravante de los trastornos mentales, de tal manera que el ministerio de la salud recomienda la realización de investigaciones que contribuyan a la identificación y tratamiento de dicho fenómeno. El presente estudio tuvo como objetivo realizar una adaptación transcultural de la Escala de Estigma para el portugués brasileño y verificar sus parámetros psicométricos preliminares. El método fue estructurado en cinco etapas: 1. obtención del permiso para adaptación; 2. traducción del instrumento; 3. síntesis y evaluación por especialistas; 4. retrotraducción y evaluación por especialistas; 5 . estudio piloto. La versión brasileña de la Escala de Estigma fue aplicada a 23 pacientes psiquiátricos. El instrumento presentó buenas evidencias de validez de contenido $(k=0,81)$ y consistencia interna $(\alpha=0,87)$. Los participantes del estudio piloto no reportaron dificultades de comprensión de los ítems. Aparte, los autores originales de la Escala de Estigma aprobaron la versión brasileña del instrumento.

Palabras clave: traducción; validación; estigma de la enfermedad mental; salud mental.

O estigma é utilizado para definir características e/ ou comportamentos que diferem daqueles exibidos pela maioria dos indivíduos que compõem uma sociedade, sendo comum que pessoas que apresentem características consideradas atípicas sejam depreciadas e em alguns casos excluídas do meio cultural no qual estão inseridas. Observa-se assim, que o estigma é um fenômeno social, não sendo inerente apenas a um indivíduo ou grupo de pessoas (Corrigan \& Watson, 2002; Goffman, 1988; Regnerus, 2017).

Endereço para correspondência: Avenida Ipiranga, 6681, Prédio 11, $9^{\circ}$ andar, sala 322, Partenon, 90619-900, Porto Alegre, RS. Tel.: (51) $3320-3683$. E-mail: nicolas.deoliveira@hotmail.com

Artigo derivado da 'Dissertação de mestrado' de 'Nicolas de Oliveira Cardoso' com orientação de 'Irani Iracema de Lima Argimon', defendida em '2019' no programa de pós-graduação 'em psicologia’ da 'Pontifícia Universidade Católica do Rio Grande do Sul. 
Inúmeros estigmas estão presentes na sociedade brasileira, principalmente contra minorias (Magno, Dourado, \& Silva, 2018), pessoas com diagnóstico de HIV (Garbin, Martins, Belila, \& Garbin, 2017) ou de doença mental (Weber \& Juruena, 2017). O EDM é considerado um fator agravante dos quadros psicopatológicos (Gumus, Dikec, \& Ergun, 2017; Weber \& Juruena, 2017). O estigma da doença mental (EDM) pode ser dividido em quatro categorias: 1. estigma internalizado; 2. estigma antecipado; 3 . estigma experienciado; e 4. estigma percebido.

O estigma internalizado está relacionado à absorção dos estereótipos, preconceitos e discriminações sociais ao self (si próprio) (Corrigan \& Watson, 2002; Fox, Earnshaw, Taverna, \& Vogt, 2017). O estigma antecipado pode ser compreendido como a expectativa de ser vítima de estereótipos, preconceito ou discriminação (Bos, Pryor, Reeder, \& Stutterheim, 2013; Fox et al., 2017). Já o estigma experienciado é constituído por meio de situações, durante o decorrer do ciclo vital, nas quais uma pessoa foi discriminada em decorrência de seu diagnóstico de transtorno mental (Fox et al., 2017; Wahl, 1999). Por fim, o estigma percebido é uma característica presente tanto no estigmatizador como no estigmatizado e é definido como a percepção social dos estereótipos, preconceitos e discriminações sociais contra pessoas com doença mental (Bos et al., 2013; Fox et al., 2017).

Uma revisão sistemática recente constatou a existência de mais de 400 instrumentos que se propõem a medir o EDM. Sendo que dois terços dessas escalas não foram submetidas a processos de validação. Além disso, boa parte das escalas restantes tem por foco a mensuração do estigma internalizado ou o estigma característico de um transtorno mental específico (e.g., depressão), ou avaliam a percepção da comunidade e profissionais da área da saúde sobre o EDM. Poucos são os instrumentos com enfoque no EDM percebido e experienciado por pessoas que vivem com um transtorno mental (Fox et al., 2017). Um dos instrumentos conhecidos por avaliar a percepção das experiências vivenciadas por pessoas com doença mental é a The Stigma Scale (Fox et al., 2017; King et al., 2007).

A The Stigma Scale foi desenvolvida na Inglaterra por King et al. (2007) em uma amostra composta por 193 pacientes ambulatoriais e em internação psiquiátrica. Sua primeira versão apresentava 42 questões relacionadas ao EDM, essas questões foram formuladas levando em consideração os sentimentos e as experiências de 46 pessoas com doença mental entrevistadas em um estudo qualitativo conduzido previamente pelos mesmos autores (Dinos, Stevens, Serfaty, Weich, \& King, 2004).

Em relação às propriedades psicométricas do instrumento, os autores da The Stigma Scale inicialmente mensuraram a confiabilidade dos 42 itens por meio de teste-reteste e removeram sete itens com $\kappa$ inferior a 0,4 . Uma análise fatorial foi realizada com os 35 itens restantes na qual foram identificados três fatores e outros sete itens foram excluídos por apresentarem carga fatorial inferior a 0,4 em qualquer um dos fatores. $O$ primeiro fator (Discriminação, $\alpha=0,87$ ) é composto por 13 itens que discorrem sobre a capacidade de perceber as atitudes preconceituosas de outras pessoas e sobre as experiências de vida onde foi vítima de discriminação devido ao diagnóstico de transtorno mental. Exemplos de itens: 1) Eu fui discriminado durante os estudos devido aos meus problemas de saúde mental; 2) Às vezes sinto que estão falando de mim devido aos meus problemas de saúde mental (King et al., 2007).

O segundo fator (Revelação, $\alpha=0,85$ ) é composto por 10 itens que avaliam a percepção das pessoas com doença mental frente à possibilidade de revelar, aos amigos, familiares ou membros da comunidade, que possuem diagnóstico de transtorno mental. Exemplos de itens: 14. Eu não me importo que as pessoas da minha vizinhança saibam que já tive problemas de saúde mental; 27. Eu sinto a necessidade de esconder meus problemas de saúde mental dos meus amigos. O terceiro fator (Aspectos positivos, $\alpha=0,64)$ é composto por cinco itens relacionados aos possíveis pontos positivos de uma doença mental. Exemplos de itens: 10. Meus problemas de saúde mental me fizeram aceitar melhor as outras pessoas; 23 . Ter tido problemas de saúde mental me fez uma pessoa mais forte. A versão final da The Stigma Scale é composta por 28 itens e apresenta consistência interna de $\alpha=0,87$ (King et al., 2007).

A The Stigma Scale se destaca por ter sido adaptada para mais de sete países, apresentando boas evidências de consistência interna tanto em sua versão original quanto em suas adaptações transculturais. Contudo, duas exceções foram observadas nos estudos de Ho et al. (2015) e Morandi et al. (2013) onde houve necessidade de redução no número de itens das adaptações devido à obtenção de baixos índices de consistência interna.

Algumas das principais críticas durante a validação original da The Stigma Scale estão relacionadas à ausência de controle das variáveis que podem influenciar nos escores de EDM, como a intensidade dos sintomas (Haghighat, 2007) e possíveis diferenças entre pacientes ambulatoriais e em internação psiquiátrica visto que, pacientes em internação podem apresentar maior sintomatologia a ponto de interferir no juízo crítico (e.g., delírios e alucinações) ou sofrerem discriminação dos profissionais de saúde (Haghighat, 2007; Mascayano et al., 2016). Os próprios autores da The Stigma Scale sugeriram a realização de novos estudos para avaliação do instrumento em diferentes características clínicas e demográficas, em especial as diferenças entre sexo, etnia e entre indivíduos com e sem sintomas característicos de transtorno mental (King et al., 2007). No entanto, a maior parte das adaptações $(n=4)$ não avaliou a presença de sintomas psiquiátricos durante a mensuração do EDM e validação do instrumento. Talvez uma possível solução para essa situação seja a realização de analises de funcionamento diferencial dos itens (differential item functioning, DIF) (Teresi, 2001), para identificar possíveis diferenças nos parâmetros psicométricos dos itens em 
função do tipo de respondente (e.g., pacientes internados e ambulatoriais, diferença entre severidade dos sintomas) (Teresi, 2001). Uma vez identificadas as diferenças, elas demandariam a produção de tabelas normativas por grupos. Destaca-se que, até onde se tem conhecimento, esse processo não costuma ser realizado nas adaptações desse instrumento (Meier, Csiernik, Warner, \& Forchuk, 2015; Morandi et al., 2013; Reynoso, Dávalos, \& García, 2011; Sarkin et al., 2015).

A literatura também sugere que o diagnóstico pode influenciar os escores do EDM. Contudo, não há um consenso sobre qual patologia é mais estigmatizada, quando comparadas, existem estudos que apontam para os transtornos de humor (Corker et al., 2014; Picco et al., 2017) e outros para os transtornos psicóticos (Ilic et al., 2013; Sarkin et al., 2015). Independentemente de qual seja o diagnóstico mais estigmatizado, sabe-se que a Agenda Nacional de Prioridades de Pesquisa em Saúde (ANPPS) aponta o EDM como um dos principais enfoques para estudo no campo da saúde mental brasileira (Brasil, 2015).

Essa preocupação ocorre devido à redução da busca por tratamento psicológico e a não adesão dos pacientes a este, os quais podem parar de tomar as medicações ou frequentar as sessões de psicoterapia devido ao EDM presente na sociedade (Corrigan, Druss, \& Perlick, 2014; Fox et al., 2017). Considerando esse cenário, assim como a carência de instrumentos para mensuração da percepção e das experiências relacionadas ao EDM no Brasil, este estudo teve como objetivo realizar a adaptação transcultural da The Stigma Scale, para o português brasileiro e verificar seus parâmetros psicométricos preliminares.

No presente estudo será investigada a fidedignidade do instrumento por meio da análise de concordância entre juízes e consistência interna. Espera-se a obtenção de níveis de concordância entre juízes superiores a $\kappa \geq 0.6 \mathrm{e}$ níveis de fidedignidade $\alpha \geq 0.70$.

\section{Método}

Os procedimentos utilizados para a adaptação transcultural da The Stigma Scale seguiram as recomendações de Guillemmin, Bombardier e Beaton (1993) e da International Test Comission (ITC, 2017). A Figura 1 apresenta um fluxograma composto pelos passos utilizados para a adaptação transcultural da The Stigma Scale, que serão expostos nas seções seguintes.

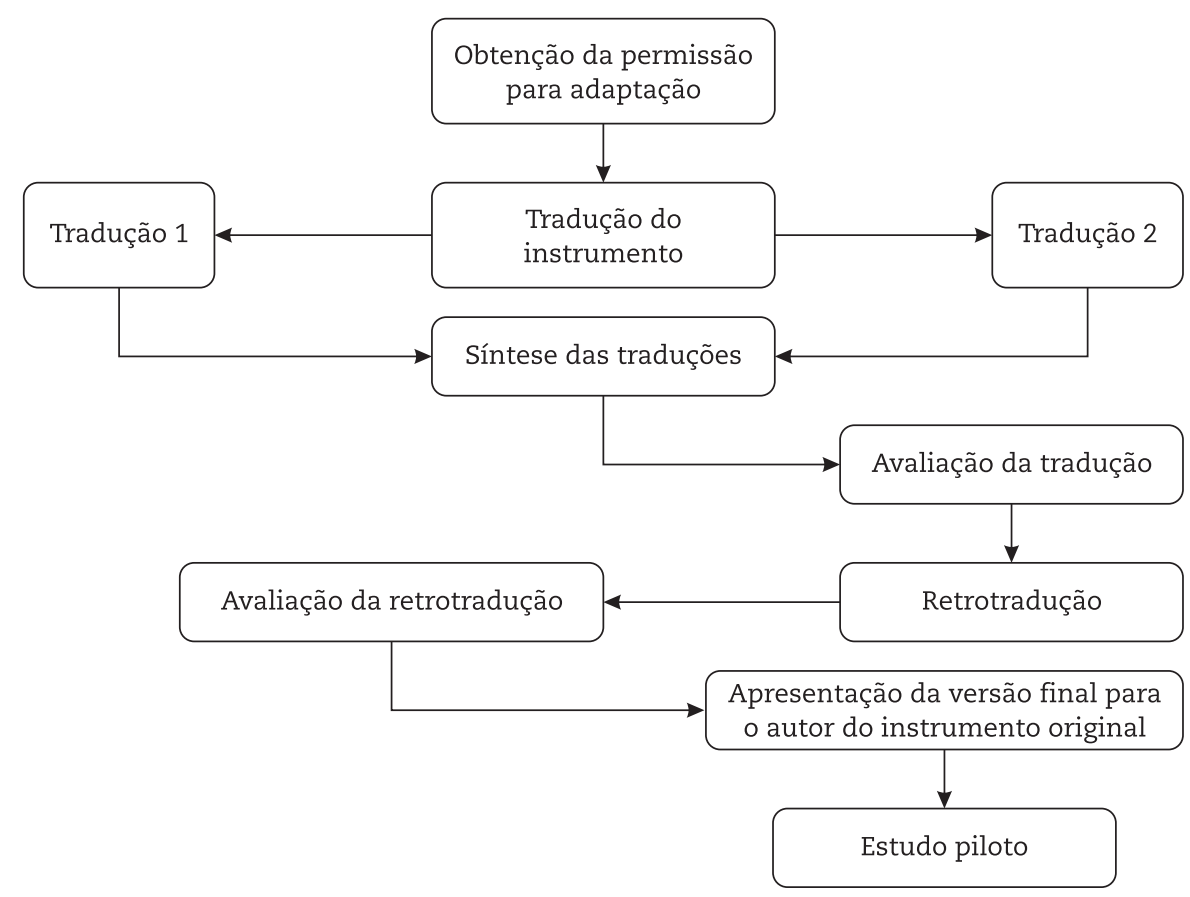

Figura 1. Procedimentos para Adaptação Transcultural da The Stigma Scale

\section{Obtenção da permissão para adaptação}

O processo de adaptação transcultural da The Stigma Scale teve início após a obtenção da autorização dos dois primeiros autores do instrumento (King et al., 2007). Os autores permitiram a adaptação e solicitaram que a versão retrotraduzida fosse enviada para sua apreciação. Dessa forma, além dos seis passos seguidos baseados nos modelos de Guillemmin et al. (1993) e da ITC (2017) também realizamos outra etapa, antes da realização do estudo piloto, de envio da versão retrotraduzida aos autores do instrumento. 


\section{Tradução do instrumento}

Foram realizadas duas traduções independentes da versão original em inglês da The Stigma Scale para o português brasileiro. Ambos os tradutores eram bilíngues, um psicólogo nativo brasileiro com proficiência em língua inglesa e conhecimento técnico na área do EDM e por outro tradutor nativo canadense com proficiência em português e sem conhecimento técnico do vocabulário da área investigada.

\section{Síntese e avaliação por especialistas}

A síntese das duas traduções foi realizada por uma psicóloga com experiência na construção de medidas psicológicas. Foi levada em consideração a equivalência semântica, idiomática e conceitual, assim como a utilização de linguagem mais simples, a qual possibilitaria a compreensão do instrumento em populações com baixa escolaridade. A versão unificada foi avaliada por três especialistas com experiência na construção de medidas psicológicas.

\section{Retrotradução e avaliação por especialistas}

A versão final da escala em português, após realização das modificações sugeridas pelos especialistas, foi traduzida para o inglês por um nativo australiano com proficiência em português sem conhecimento técnico da área investigada. A versão retrotraduzida foi comparada com a versão original do instrumento por outro especialista com experiência na construção de medidas psicológicas, o qual não participou em nenhuma das etapas anteriores da adaptação. Essa versão também foi avaliada por um dos autores da versão original do instrumento e pelos autores do presente estudo.

\section{Estudo piloto}

Os autores deste estudo elaboraram uma figura de suporte (apêndice A) com as cinco opções de resposta da The Stigma Scale a qual poderá ser entregue aos participantes que não desejarem ou estiverem impossibilitados (e.g., lesão em membro superior) de preencher o instrumento. Nesses casos, o entrevistador poderá ler o instrumento ao participante e assinalar a alternativa indicada por ele. Essa figura foi cogitada, pois parte da amostra para investigação de evidências de validade da estrutura interna da The Stigma Scale será composta por pacientes hospitalares. Além disso, com o intuito de avaliar a clareza do formato de apresentação e linguagem do instrumento, também foi elaborado um questionário para avaliação da The Stigma Scale, pelos participantes do estudo piloto, composto por duas questões quantitativas e duas qualitativas. As questões quantitativas são referentes ao entendimento das instruções e tamanho da letra: 1. As instruções do questionário são claras e de fácil entendimento? 2. O tamanho da letra foi adequado e fácil de ser lido?

Essas questões foram mensuradas por meio de escala Likert de 0-4 onde 0 representa nada compreensível e 4 totalmente compreensível. As questões qualitativas estão relacionadas à clareza dos itens e conteúdo: 1. Alguma palavra não ficou clara para você? Qual? Tem alguma sugestão? 2. Alguma questão não ficou clara? Qual? O que não ficou claro? Tem alguma sugestão?

\section{Participantes}

Participaram deste estudo 23 adultos internados ou em tratamento ambulatorial em um hospital geral da região metropolitana de Porto Alegre, com média de idade de 39 anos $(D P=14,3)$, sendo a maioria dos participantes do sexo masculino $(n=14 ; 61 \%)$. Os diagnósticos mais prevalentes na amostra foram: o Transtorno por Uso de Álcool $n=7 ; 30,4 \%$ ), Transtorno Depressivo Maior induzido por substância e Transtorno de Ansiedade Generalizada, ambos com a mesma prevalência $(n=5$; $21,7 \%)$, seguidos pela Depressão Maior $(n=4 ; 17,3 \%)$. Embora todos os participantes fizessem uso de alguma medicação, mais da metade $(n=13 ; 56,5 \%)$ não souberam informar seu nome nem há quanto tempo a utilizavam.

Em relação à presença de doença psiquiátrica na família, pouco mais de um terço da amostra $(n=8 ; 34,7 \%)$ informou possuir pelo menos um familiar com diagnóstico psiquiátrico. Em geral, quando questionados sobre seu diagnóstico, pouco menos da metade dos participantes $(n=10 ; 43,4 \%)$ aceitavam sua condição clínica. Observou-se ainda que menos da metade da amostra concluiu o ensino médio $(n=11 ; 47,8 \%)$. A Tabela 1 exibe detalhadamente os dados sociodemográficos e de saúde da amostra.

Tabela 1

Caracterização da Amostra $(n=23)$

\begin{tabular}{|c|c|c|c|c|c|c|}
\hline ID & $\begin{array}{l}\text { Idade } \\
\text { (sexo) }\end{array}$ & Diagnóstico & Med (tempo) & $\begin{array}{l}\text { Familiar } \\
\text { c/ Diag. } \\
\text { Psiq. }\end{array}$ & $\begin{array}{l}\text { Concorda } \\
\text { c/ Diag. }\end{array}$ & Escolaridade \\
\hline ip01 & $39(\mathrm{~m})$ & $\begin{array}{l}\text { tr. depressivo persistente c/ } \\
\text { sintomas psicóticos }\end{array}$ & $\mathrm{n} / \mathrm{s}$ & $\operatorname{sim}$ & $\operatorname{sim}$ & $\begin{array}{l}\text { médio } \\
\text { incompleto }\end{array}$ \\
\hline ip02 & $34(\mathrm{~m})$ & $\begin{array}{l}\text { tr. por uso de cocaína/crack } \\
\text { tr. por uso de álcool } \\
\text { tr. de ansiedade generalizada }\end{array}$ & $\mathrm{n} / \mathrm{s}$ & $\operatorname{sim}$ & $\operatorname{sim}$ & médio completo \\
\hline
\end{tabular}


Tabela 1 (continuação)

Caracterização da Amostra $(n=23)$

\begin{tabular}{|c|c|c|c|c|c|c|}
\hline ID & $\begin{array}{l}\text { Idade } \\
\text { (sexo) }\end{array}$ & Diagnóstico & Med (tempo) & $\begin{array}{l}\text { Familiar } \\
\text { c/ Diag. } \\
\text { Psiq. }\end{array}$ & $\begin{array}{l}\text { Concorda } \\
\text { c/ Diag. }\end{array}$ & Escolaridade \\
\hline ip07 & $56(\mathrm{f})$ & $\begin{array}{l}\text { tr. depressivo persistente } \\
\text { tr. de ansiedade generalizada }\end{array}$ & $\mathrm{n} / \mathrm{s}$ & não & $\operatorname{sim}$ & $\begin{array}{l}\text { fundamental } \\
\text { completo }\end{array}$ \\
\hline ip11 & $21(\mathrm{~m})$ & $\begin{array}{l}\text { tr. por uso de cannabis } \\
\text { tr. psicótico induz. subst. }\end{array}$ & $\begin{array}{l}\text { haldol, lítio, sertralina } \\
\text { (21 dias) }\end{array}$ & não & $\operatorname{sim}$ & médio completo \\
\hline ip12 & $52(\mathrm{~m})$ & $\begin{array}{l}\text { tr. depressivo maior c/ sintomas } \\
\text { psicóticos } \\
\text { tr. de ansiedade generalizada }\end{array}$ & $\begin{array}{l}\text { lítio carbamazepina } \\
\text { donaren } \\
\text { (6 anos) }\end{array}$ & $\operatorname{sim}$ & $\operatorname{sim}$ & $\begin{array}{l}\text { fundamental } \\
\text { completo }\end{array}$ \\
\hline ip13 & $58(\mathrm{~m})$ & $\begin{array}{l}\text { tr. depressivo maior passado } \\
\text { induz. subst. } \\
\text { tr. por uso álcool }\end{array}$ & $\begin{array}{l}\text { diazepan } \\
\text { (38 anos) }\end{array}$ & não & mss & médio completo \\
\hline ip14 & 19(f) & $\begin{array}{l}\text { tr. depressivo maior } \\
\text { tr. de personalidade borderline }\end{array}$ & $\begin{array}{l}\text { fluoxetina } \\
\text { (7 dias) }\end{array}$ & não & não & $\begin{array}{l}\text { médio } \\
\text { incompleto }\end{array}$ \\
\hline ip15 & $31(\mathrm{~m})$ & $\begin{array}{l}\text { tr. psicótico induz. subst. } \\
\text { tr. depressivo maior passado } \\
\text { induz. subst. } \\
\text { tr. de ansiedade generalizada } \\
\text { induz. subst. }\end{array}$ & $\mathrm{n} / \mathrm{s}$ & não & mss & $\begin{array}{l}\text { fundamental } \\
\text { completo }\end{array}$ \\
\hline ip18 & $40(f)$ & $\begin{array}{l}\text { tr. depressivo maior passado } \\
\text { induz. subst. } \\
\text { tr. por uso de álcool; tept }\end{array}$ & $\begin{array}{l}\text { fluoxetina } \\
\text { (7 dias) }\end{array}$ & $\operatorname{sim}$ & $\operatorname{sim}$ & $\begin{array}{l}\text { fundamental } \\
\text { incompleto }\end{array}$ \\
\hline ip26 & $56(f)$ & $\begin{array}{l}\text { tr. depressivo maior; tept } \\
\text { tr. de ansiedade generalizada } \\
\text { induz. subst. }\end{array}$ & $\mathrm{n} / \mathrm{s}$ & $\operatorname{sim}$ & não & $\begin{array}{l}\text { fundamental } \\
\text { incompleto }\end{array}$ \\
\hline ip27 & $23(f)$ & $\begin{array}{l}\text { tr. depressivo maior passado } \\
\text { toc }\end{array}$ & $\mathrm{n} / \mathrm{s}$ & $\operatorname{sim}$ & $\operatorname{sim}$ & $\begin{array}{l}\text { superior } \\
\text { incompleto }\end{array}$ \\
\hline ip28 & $42(\mathrm{~m})$ & tr. por uso de álcool & $\mathrm{n} / \mathrm{s}$ & não & mss & não alfabetizado \\
\hline ip29 & $42(\mathrm{~m})$ & tr. por uso de álcool & $\mathrm{n} / \mathrm{s}$ & não & $\mathrm{n} / \mathrm{s}$ & médio completo \\
\hline ip30 & $53(\mathrm{~m})$ & $\begin{array}{l}\text { tr. por uso de álcool } \\
\text { tr. depressivo maior induz. subst. }\end{array}$ & $\mathrm{n} / \mathrm{s}$ & não & mss & médio completo \\
\hline ip31 & $20(f)$ & anorexia & $\mathrm{n} / \mathrm{s}$ & não & $\mathrm{n} / \mathrm{s}$ & médio completo \\
\hline ip32 & $26(\mathrm{~m})$ & $\begin{array}{l}\text { tr. delirante atual } \\
\text { tept }\end{array}$ & haldol (n/s) & não & $\mathrm{n} / \mathrm{s}$ & $\begin{array}{l}\text { fundamental } \\
\text { completo }\end{array}$ \\
\hline ip33 & $22(\mathrm{~m})$ & tr. uso de cocaína & $\mathrm{n} / \mathrm{s}$ & não & mss & $\begin{array}{l}\text { fundamental } \\
\text { completo }\end{array}$ \\
\hline ip34 & $58(\mathrm{~m})$ & tr. por uso de álcool & $\mathrm{n} / \mathrm{s}$ & não & $\operatorname{sim}$ & $\begin{array}{l}\text { fundamental } \\
\text { incompleto }\end{array}$ \\
\hline ip35 & $45(\mathrm{f})$ & $\begin{array}{l}\text { tr. depressivo atual induz. subst. } \\
\text { tr. por uso de ansiolítico } \\
\text { tr. de ansiedade generalizada } \\
\text { induz. subst. }\end{array}$ & $\mathrm{n} / \mathrm{s}$ & não & mss & $\begin{array}{l}\text { médio } \\
\text { incompleto }\end{array}$ \\
\hline amb01 & $61(\mathrm{~m})$ & $\begin{array}{l}\text { tr. depressivo maior atual } \\
\text { tr. de ansiedade generalizada }\end{array}$ & rivotril $(\mathrm{n} / \mathrm{s})$ & $\operatorname{sim}$ & não & $\begin{array}{l}\text { superior } \\
\text { incompleto }\end{array}$ \\
\hline amb02 & 19(f) & tdah & ritalina (4 anos) & não & $\operatorname{sim}$ & $\begin{array}{l}\text { superior } \\
\text { incompleto }\end{array}$ \\
\hline
\end{tabular}


Tabela 1 (continuação)

Caracterização da Amostra (n=23)

\begin{tabular}{|c|c|c|c|c|c|c|}
\hline ID & $\begin{array}{l}\text { Idade } \\
\text { (sexo) }\end{array}$ & Diagnóstico & Med (tempo) & $\begin{array}{c}\text { Familiar } \\
\text { c/ Diag. } \\
\text { Psiq. }\end{array}$ & $\begin{array}{l}\text { Concorda } \\
\text { c/ Diag. }\end{array}$ & Escolaridade \\
\hline amb03 & 39(f) & $\begin{array}{l}\text { tr. de ansiedade generalizada } \\
\text { tdah }\end{array}$ & $\begin{array}{l}\text { venvanse } \\
\text { (5 anos) }\end{array}$ & $\operatorname{sim}$ & $\operatorname{sim}$ & $\begin{array}{l}\text { doutorado } \\
\text { incompleto }\end{array}$ \\
\hline amb04 & 43(m) & esquizofrenia & $\begin{array}{l}\text { olanzapina } \\
\text { (11 anos) }\end{array}$ & não & $\mathrm{n} / \mathrm{s}$ & médio completo \\
\hline
\end{tabular}

Nota. amb=ambulatorial; Diag=diagnóstico; f=feminino; ID=código do participante; ip=internação psiquiátrica; m=masculino; Med=medicamento; mss=missing; n/s=não sabia; Psiq=psiquiátrico; tdah=transtorno de déficit de atenção e hiperatividade; tept=transtorno de estresse pós-traumático; tr.=transtorno

\section{Instrumentos}

Questionário de dados sociodemográficos e de saúde. Esse questionário contemplou variáveis relacionadas à idade, sexo, estado civil, escolaridade, nível socioeconômico, saúde mental e uso de medicamentos.

Entrevista Clínica Estruturada para os Transtornos do DSM-5 - Versão Clínica (SCID-5$C V$; First, Williams, Karg, \& Spitzer, 2015). A SCID5-CV foi utilizada para confirmar a presença de algum transtorno mental específico, ou a ausência destes. A SCID-5-CV é um guia de entrevista para a realização do diagnóstico dos transtornos psiquiátricos encontrados no contexto clínico. O aplicador foi treinado para seu uso. $\mathrm{O}$ instrumento foi desenhado para ser administrado por qualquer profissional da área de saúde.

The Stigma Scale (King et al., 2007). A escala é composta por 28 questões que avaliam o estigma percebido e experienciado por indivíduos com diagnóstico de transtorno mental, é autoaplicável e leva entre 5-10 minutos para ser preenchida. A The Stigma Scale apresenta opções de resposta em escala Likert, variando entre 0-4 na direção de maior EDM. Esses números são representados da seguinte forma: 0 - discordo plenamente; 1 - discordo; 2 - não concordo e nem discordo; 3 - concordo; e 4 - concordo plenamente. Alguns itens do instrumento devem ser avaliados de forma reversa e estão sinalizados com a letra "(D)", logo, apenas a soma do escore total (i.e., sem reversão) obtido no instrumento não é suficiente para mensurar se o participante apresenta alto índice de EDM. Os 28 itens da The Stigma Scale estão divididos em três subescalas: 1. discriminação; 2. revelação; e 3. aspectos positivos. A subescala 1 (itens $1,2,8,9,11,13,17,18,19,20,21,22$ e 26) avalia a percepção de possíveis situações onde o indivíduo possa ter sido discriminado em decorrência de sua doença mental. A subescala 2 (itens 4, 5, 12, 14, 15, 16, 24, 25, 27 e 28) mensura a percepção e as experiências das pessoas com doença mental frente a revelação de seu diagnóstico a outras pessoas. A subescala 3 (itens 3, 6, 7, 10 e 23) investiga os possíveis pontos positivos de uma doença mental.

\section{Procedimentos de coleta}

Todos os dados foram obtidos de adultos entre os 18 e 61 anos fluentes em português brasileiro, com diagnóstico de transtorno mental, que manifestaram interesse em participar da pesquisa por meio da assinatura do Termo de Consentimento Livre e Esclarecido (TCLE). Os participantes foram selecionados por conveniência. Visando avaliar a clareza da linguagem do instrumento a ser adaptado, após o preenchimento dos instrumentos da pesquisa, os participantes foram divididos em dois grupos: 1. participantes em internação psiquiátrica; 2 . participantes ambulatoriais.

1. Participantes em internação psiquiátrica - composto por indivíduos internados em uma unidade psiquiátrica de um hospital geral da região metropolitana de Porto Alegre.

2. Participantes ambulatoriais - composto por indivíduos com diagnóstico de transtorno mental residentes da região metropolitana de Porto Alegre.

Foram excluídos os participantes que não concluíram todas as medidas e procedimentos planejados $(n=16)$. Todos os participantes excluídos faziam parte do grupo IP, o qual era composto originalmente por 35 participantes, o que caracteriza uma perda amostral de $45,7 \%$, visto que apenas 19 participantes desse grupo responderam a todos os questionários propostos. Tal perda amostral está relacionada principalmente a alta dos participantes da internação psiquiátrica antes da aplicação de todos os testes.

\section{Análise de dados}

Todas as análises foram realizadas por meio do programa estatístico R versão 3.5.1 (R Core Team, 2018). Evidências de validade de conteúdo foram obtidas considerando a representatividade dos itens, por meio da avaliação dos juízes e estudo piloto. Os juízes/ especialistas que participaram da adaptação do instrumento receberam por e-mail o instrumento traduzido e o devolveram com sugestões de alterações dos itens e título da escala. 
O índice de confiabilidade entre avaliadores foi mensurado por meio do índice Light's Kappa, o qual foi desenvolvido para análise da concordância entre três ou mais avaliadores, sendo a análise mais recomendada para estes casos (Hallgren, 2012; Light, 1971). A análise da confiabilidade entre os avaliadores, por meio do índice Light's Kappa, foi realizada no software R por meio da instalação e utilização do pacote de dados IRR (Gamer, Lemon, Fellows, \& Singh, 2015).

Após a adaptação da escala, o projeto foi apresentado à direção de um hospital geral da região metropolitana de Porto Alegre. A partir da autorização do hospital e do Comitê de Ética em Pesquisa (CEP) da Universidade Luterana do Brasil (ULBRA) sob o CAAE $\mathrm{n}^{\circ}$ 70815317.0.0000.5349, indivíduos em internação psiquiátrica em um hospitalar geral ou em tratamento ambulatorial residentes na região metropolitana de Porto Alegre foram convidados a participar do estudo. A consistência interna da versão final da The
Stigma Scale (e subescalas), traduzida e adaptada para o português brasileiro, foi estimada por meio de matrizes de correlações policóricas dos itens e do coeficiente alfa de Cronbach ordinal (Zumbo, Gadermann, \& Zeisser, 2007), ambas as equações foram computadas no software R por meio da utilização do pacote de dados Psych (Revelle, 2018).

\section{Resultados}

Após a realização das traduções da The Stigma Scale e obtenção da versão sintetizada do instrumento, a escala foi submetida à três especialistas independentes. Estes sugeriram modificações relacionadas à equivalência semântica e idiomática de algumas expressões presentes nos itens do instrumento. A Tabela 2 exibe os itens da versão sintetizada em português brasileiro, as sugestões de modificações dos especialistas e a versão adaptada e enviada para retrotradução após a apreciação das sugestões.

Tabela 2

Sugestões dos Especialistas Durante a Adaptação Transcultural da The Stigma Scale

\begin{tabular}{lll}
\hline \multicolumn{1}{c}{ Versão sintetizada } & \multicolumn{1}{c}{ Versão enviada para retrotradução } \\
\cline { 1 - 1 } $\begin{array}{ll}\text { Escala de Estigma } \\
\text { saúde mental. }\end{array}$ & & $\begin{array}{l}\text { Escala de estigma da doença mental discriminado durante os } \\
\text { estudos... }\end{array}$
\end{tabular}

2. Às vezes sinto que estão falando de mim devido aos meus problemas de saúde mental.

Mantido

3. Ter tido problemas de saúde mental me tornou uma pessoa mais compreensiva.

4. Eu não me sinto mal por ter tido problemas de saúde mental.

Mantido

5. Tenho receio de dizer às pessoas que recebo tratamento psicológico.

6. Algumas pessoas com problemas de saúde mental são perigosas.

7. As pessoas têm sido compreensivas com meus problemas de saúde mental.

8. Fui discriminado pela polícia por causa dos meus problemas de saúde mental.

9. Fui discriminado por meus empregadores por causa dos meus problemas de saúde mental.

10. Meus problemas de saúde mental me fizeram aceitar melhor as outras pessoas.

11. Muitas vezes eu me sinto sozinho por causa dos meus problemas de saúde mental.

12. Tenho medo de como outras pessoas irão reagir se descobrirem sobre meus problemas de saúde mental.

Mantido

Tenho receio de dizer às pessoas que faço tratamento psicológico

Mantido

Mantido

Mantido

Fui discriminado por meus chefes...

Mantido

Mantido

Mantido

Mantido

Mantido

14. Eu não me importo que as pessoas da minha vizinhança saibam que já tive problemas de saúde mental. 
Tabela 2 (continuação)

Sugestões dos Especialistas Durante a Adaptação Transcultural da The Stigma Scale

\begin{tabular}{lll}
\hline Versão sintetizada & Versão enviada para retrotradução \\
\cline { 1 - 1 } & Escala de Estigma & Escala de estigma da doença mental \\
\hline
\end{tabular}

15. Eu diria que tive problemas de saúde mental se estivesse em uma entrevista de emprego.

16. Tenho receio de dizer às pessoas que tomo medicamentos para problemas de saúde mental.

Mantido

Mantido

17. A reação das pessoas aos meus problemas de saúde mental fazem com que eu me isole.

18. Eu estou com raiva da maneira que as pessoas reagiram aos meus problemas de saúde mental.

19. Eu não tive qualquer problema com as pessoas devido aos meus problemas de saúde mental.

20. Eu fui discriminado por profissionais de saúde devido aos meus problemas de saúde mental.

21. Pessoas me evitaram por causa dos meus problemas de saúde mental.

22. Pessoas me insultaram por causa dos meus problemas de saúde mental.

23. Ter tido problemas de saúde mental me fez uma pessoa mais forte.

24. Eu não me sinto constrangido por causa dos meus problemas de saúde mental.

25. Eu evito contar às pessoas sobre os meus problemas de saúde mental.

Mantido

Eu sinto raiva...

Mantido

Já me senti discriminado...

Já me evitaram por causa dos meus problemas de saúde mental

Já me insultaram por causa dos meus problemas de saúde mental

Mantido

Mantido

Mantido

26. Ter tido problemas de saúde mental me faz sentir que a vida é injusta.

Mantido

Mantido

27. Eu sinto a necessidade de esconder meus problemas de saúde mental dos meus amigos.

28. Eu acho difícil dizer às pessoas que tenho problemas de saúde mental.

Mantido

O nível de concordância entre os especialistas, obtido por meio do índice Light's Kappa (Apêndice B), foi de 0,81, com escore $z=1,98$, e $p \leq 0,05$ o que indica concordância substancial entre as avaliações dos especialistas. Sendo assim, a Escala de Estigma da Doença Mental (EEDM) manteve os 28 itens de sua versão original. Contudo, com o intuito de garantir a equivalência semântica e idiomática dos itens no contexto brasileiro, alguns itens foram reformulados conforme sugerido pelos especialistas.

Posteriormente, foi realizada a retrotradução, por um nativo australiano sem conhecimento técnico do vocabulário da área de estudo. Não foram constatadas diferenças significativas entre a versão original da The Stigma Scale e a retrotraduzida. Sendo assim, não houve sugestões de alterações pelos avaliadores da versão retrotraduzida ficando essa versão estabelecida para realização do estudo piloto.

\section{Avaliação do estudo piloto}

Em relação à compreensão verbal, todos os participantes do grupo $\mathrm{AMB}$ responderam ao questionário para avaliação da EEDM formulado pelos autores deste estudo. Nenhum dos participantes relatou dúvidas ou dificuldades relacionadas ao entendimento do conteúdo do instrumento. Alguns dos participantes em IP $(n=6)$ responderam a esse questionário, sendo que dois sinalizaram a necessidade de aumentar o tamanho da fonte utilizada (Times New Roman, 10). Nenhum dos seis participantes relatou dúvidas ou dificuldades relacionadas ao entendimento do conteúdo do instrumento. A Tabela 3 apresenta a avaliação preliminar da consistência interna da EEDM e comparação com sua versão original e adaptações subsequentes que mantiveram os 28 itens originais do instrumento. 
Tabela 3

Consistência Interna da EEDM e Comparação com sua Versão Original e outras Adaptações

\begin{tabular}{lcccc}
\hline \multicolumn{1}{c}{ Consistência interna } & $\begin{array}{c}\text { Subescala de } \\
\text { discriminação } \\
\text { (13 itens) }\end{array}$ & $\begin{array}{c}\text { Subescala de } \\
\text { revelação } \\
\text { (10 itens) }\end{array}$ & $\begin{array}{c}\text { Subescala de } \\
\text { aspectos positivos } \\
\text { (5 itens) }\end{array}$ & $\begin{array}{c}\text { Total } \\
\text { (28 itens) }\end{array}$ \\
\hline Brasil (presente estudo) & 0,80 & 0,78 & 0,58 & 0,87 \\
Japão (Mizuno et al., 2017) & 0,84 & 0,86 & 0,82 & 0,89 \\
Canada (Meier et al., 20151) & 0,83 & 0,79 & 0,46 & 0,86 \\
EUA (Sarkin et al., 2014) & 0,88 & 0,85 & 0,52 & 0,87 \\
México (Reynoso et al., 2011) & 0,83 & 0,76 & 0,60 & 0,87 \\
Inglaterra/Original (King et al., 2007) ${ }^{1}$ & 0,87 & 0,85 & 0,64 & 0,87 \\
\hline
\end{tabular}

Nota. ${ }^{1} \alpha$ de Cronbach; ${ }^{2} \Omega ;{ }^{3} \alpha$ Ordinal

\section{Discussão}

Este estudo objetivou apresentar as etapas de adaptação transcultural e as primeiras evidências de parâmetros psicométricos da EEDM. Foram cumpridas as etapas iniciais para o fornecimento de um novo instrumento psicométrico capaz de mensurar o EDM em população brasileira. Embora a amostra deste estudo seja menor, por se tratar de um estudo piloto, do que as utilizadas pelas demais adaptações expostas na Tabela 3, observa-se semelhança nos níveis de consistência interna obtidos entre as adaptações. Em especial com os resultados obtidos por Reynoso, Dávalos e García (2011).

Esses resultados sugerem que a versão final da EEDM (Apêndice C) apresenta consistência interna, preliminar, satisfatória em brasileiros com diagnóstico de transtorno mental (Apêndice D). Destaca-se que embora alterações relacionadas a algumas expressões britânicas, pouco ou não utilizadas no Brasil, tenham sido realizadas, poucos itens foram modificados $(n=8)$. Tal fato reforça a similaridade da versão brasileira da EEDM com sua versão original (King et al., 2007).

Ressalta-se que este artigo apresenta apenas evidências preliminares de validade da EEDM, sendo assim, outros estudos, que contemplem um número maior de participantes, e ampliem as evidências de validade apresentadas neste estudo, devem ser conduzidos. Visto que alguns autores destacam que a adaptação transcultural representa as primeiras etapas do processo de validação de um instrumento psicométrico (Borsa, Damásio, \& Bandeira, 2012; Guillemmin et al. 1993; Urbina, 2007). Além disso, existe um consenso de que não há um número de evidências de validade ou mesmo de testes estatísticos específicos os quais devam ser utilizados para que determinado instrumento seja considerado válido. Porém, sabe-se que quanto maior o número de evidências psicométricas fornecidas por um instrumento, maior será a sua confiabilidade (Borsa et al., 2012; Urbina, 2007). Além do número de participantes, este estudo também apresenta como limitação, a utilização de uma amostra composta quase exclusivamente de pacientes em internação psiquiátrica. Conforme exposto anteriormente, é importante que estudos sejam realizados levando em consideração possíveis diferenças entre amostras clínicas (Haghighat, 2007; King et al., 2007).

Ademais, destaca-se que uma revisão sistemática realizada com estudos sobre o EDM na América Latina evidenciou que os profissionais da saúde brasileiros apresentam menos atitudes negativas, perante indivíduos com transtorno mental, quando comparados com profissionais de outros países europeus (e.g., Suíça). No entanto, observou-se que o EDM advindo da família e do meio social é mais comum nos países da América Latina (e.g., Argentina, Brasil, México, Venezuela) (Mascayano et al., 2016). Em relação aos instrumentos de medida do EDM utilizados pelos estudos incluídos na revisão, observa-se que os estudos com amostras de indivíduos brasileiros com doença mental normalmente utilizam entrevistas e vinhetas clínicas (Martin, Andreoli, Pinto, \& Barreira, 2011; Peluso \& Blay, 2011) ou questionários desenvolvidos especificamente para suas amostras (Loch, 2012).

Observa-se assim a necessidade de realização de estudos brasileiros que investiguem os impactos das percepções e das experiências de EDM, dos indivíduos com doença mental, advindas dos familiares, do meio social e dos profissionais de saúde por meio de um instrumento (i.e., EEDM) bem estabelecido na literatura e utilizado em outros países (King et al., 2007; Meier et al., 2015; Mizuno et al., 2017; Reynoso et al., 2011; Sarkin et al., 2014) o qual começa a apresentar propriedades psicométricas promissoras no Brasil. Espera-se que este estudo contribua com o campo da Avaliação Psicológica no EDM, em especial, no desenvolvimento de pesquisas que investiguem a prevalência do EDM na população brasileira, para que estratégias de intervenção, em linha com o desenvolvimento de novas políticas públicas, sejam formuladas visando atender às demandas da Agenda Nacional de Prioridades de Pesquisa em Saúde (Brasil, 2015). 


\section{Agradecimentos}

Não há menções.

\section{Financiamento}

O presente trabalho foi realizado com apoio do Conselho Nacional de Desenvolvimento Científico e Tecnológico (CNPq) e da Coordenação de Aperfeiçoamento de Pessoal de Nível Superior - Brasil (CAPES) - Código de Financiamento 001.

\section{Contribuições dos autores}

Todos os autores contribuíram substancialmente para a elaboração do delineamento da pesquisa, análise e interpretação dos dados, bem como, para a revisão textual e aprovação da versão final deste estudo. Todos os autores assumem responsabilidade pública pelo conteúdo do manuscrito.

\section{Disponibilidade dos dados e materiais}

Todos os dados e sintaxes gerados e analisados durante esta pesquisa serão tratados com total sigilo devido às exigências do Comitê de Ética em Pesquisa em Seres Humanos. Porém, o conjunto de dados e sintaxes que apoiam as conclusões deste artigo estão disponíveis mediante razoável solicitação ao autor principal do estudo.

\section{Conflito de interesses}

Os autores declaram que não há conflitos de interesses.

\section{Referências}

Brasil. (2015). Agenda Nacional de Prioridades de Pesquisa em Saúde. Recuperado de http://brasil.evipnet.org/wp-content/uploads/2017/07/ ANPPS.pdf

Borsa, J. C., Damásio, B. F., \& Bandeira, D. R. (2012). Adaptação e validação de instrumentos psicológicos entre culturas: Algumas considerações. Paidéia, 22(53), 423-432. doi: 10.1590/S0103-863X2012000300014

Bos, A. E., Pryor, J. B., Reeder, G. D., \& Stutterheim, S. E. (2013). Stigma: Advances in theory and research. Basic and Applied Social Psychology, 35(1), 1-9. doi: 10.1080/01973533.2012.746147

Corker, E. A., Beldie. A., Brain, C., Jakovljevic, M., Jarema, M., Karamustafalioglu, O., ... \& Thornicroft, G. (2014). Experience of stigma and discrimination reported by people experiencing the first episode of schizophrenia and those with a first episode of depression: The FEDORA project. International Journal of Social Psychiatry, 61(5), 438-445. doi: 10.1177/0020764014551941

Corrigan, P. W., \& Watson, A. C. (2002). The paradox of self-stigma and mental illness. Clinical Psychology: Science and Practice, 9(1), 35-53. doi: 10.1093/clipsy.9.1.35

Corrigan, P. W., Druss, B. G., \& Perlick, D. A. (2014). The Impact of Mental Illness Stigma on Seeking and Participating in Mental Health Care. Psychological Science in the Public Interest, 15(2), 37-70. doi:10.1177/1529100614531398

Dinos, S., Stevens, S., Serfaty, M., Weich, S., \& King, M. (2004). Stigma: The feelings and experiences of 46 people with mental illness: qualitative study. The British Journal of Psychiatry, 184(2), 176-181. doi: 10.1192/bjp.184.2.176

First, M., Williams, J., Karg, R., \& Spitzer, R. (2015). Clinical Interview for DSM-5 - Research Version (SCID-5 for DSM-5, Research Version; SCID-5 RV). Arlington, VA: American Psychiatric Association.

Fox, A. B., Earnshaw, V. A., Taverna, E., \& Vogt, D. (2017). Conceptualizing and measuring mental illness stigma: The mental illness stigma framework and critical review of measures. Stigma and Health, 3(4), 348-376. doi: 10.1037/sah0000104

Gamer, M., Lemon, J., Fellows, I., \& Singh, P. (2015). Various coefficients of interrater reliability and agreement. The R Project for Statistical Computing. Recuperado de https://cran.r-project.org/web/packages/irr/irr.pdf

Garbin, C. A. S., Martins, R. J., Belila, N. D. M., \& Garbin, A. J. Í. (2017). O estigma de usuários do sistema público de saúde brasileiro em relação a indivíduos HIV positivo. DST Jornal Brasileiro de Doenças Sexualmente Transmissiveis, 29(1), 12-16. Recuperado de http://www. dst.uff.br/revista29-1-2017/DST\%20v29n1_12-16_PT.pdf

Goffman, E. (1988). Estigma - notas sobre a manipulação dá identidade deteriorada. $4^{\mathrm{a}}$ ed. São Paulo: LTC.

Guillemin, F., Bombardier, C., \& Beaton, D. (1993). Cross-cultural adaptation of health-related quality of life measures: Literature review and proposed guidelines. Journal of Clinical Epidemiology, 46(12), 1417-1432. doi: 10.1016/0895-4356(93)90142-N

Gumus, F., Dikec, G., \& Ergun, G. (2017). Relations among internalized stigmatization, depressive symptom frequency and family loading in first-degree caregivers of the patients treated in the psychiatry clinic of a state hospital. Archives of Psychiatric Nursing, 31(5), 522-527. doi: 10.1016/j.apnu.2017.05.003

Haghighat, R. (2007). Measuring stigma: Comment. The British Journal of Psychiatry, 191(4), 363-364. doi: 10.1192/bjp.191.4.363a

Hallgren, K. A. (2012). Computing inter-rater reliability for observational data: An overview and tutorial. Tutorials in quantitative methods for psychology, 8(1), 23.

Ho, A. H. Y., Potash, J. S., Fong, T. C. T., Ho, V. F. L., Chen, E. Y. H., Lau, R. H., ... Ho, R. T. H. (2015). Psychometric properties of a Chinese version of the Stigma Scale: Examining the complex experience of stigma and its relationship with self-esteem and depression among people living with mental illness in Hong Kong. Comprehensive Psychiatry, 56, 198-205. doi: 10.1016/j.comppsych.2014.09.016

Ilic, M., Reinecke, J., Bohner, G., Röttgers, H. O., Beblo, T., Driessen, M., ... Corrigan, P. W. (2013). Belittled, Avoided, Ignored, Denied: Assessing Forms and Consequences of Stigma Experiences of People With Mental Illness. Basic and Applied Social Psychology, 35(1), 31-35. doi: 10.1080/01973533.2012.746619

International Test Commission (2017). International Test Commission guidelines for translating and adapting tests (second edition). Recuperado de https://www.intestcom.org/files/guideline_test_adaptation_2ed.pdf

King, M., Dinos, S., Shaw, J., Watson, R., Stevens, S., Passetti, F.,... Serfaty, M. (2007). The Stigma Scale: Development of a standardised measure of the stigma of mental illness. British Journal of Psychiatry, 190(3), 248-254. doi: 10.1192/bjp. bp.106.024638 
Light, R. J. (1971). Measures of response agreement for qualitative data: Some generalizations and alternatives. Psychological bulletin, 76(5), 365. doi: $10.1037 / \mathrm{h} 0031643$

Loch, A. A. (2012). Stigma and higher rates of psychiatric re-hospitalization: São Paulo public mental health system. Brazilian Journal of Psychiatry, 34(2), 185-192. doi: 10.1590/S1516-44462012000200011

Magno, L., Dourado, I., \& Silva, L. A. V. D. (2018). Estigma e resistência entre travestis e mulheres transexuais em Salvador, Bahia, Brasil. Cadernos de Saúde Pública, 34(5), e00135917. doi: 10.1590/0102-311X00135917

Martin, D., Andreoli, S. B., Pinto, R. M. F., \& Barreira, T. M. H. D. M. (2011). Living conditions of people with psychotic disorders living in slums in Santos, Southeastern Brazil. Revista de Saúde Pública, 45(4), 693-699. doi: 10.1590/S0034-89102011000400008

Mascayano, F., Tapia, T., Schilling, S., Alvarado, R., Tapia, E., Lips, W., \& Yang, L. H. (2016). Stigma toward mental illness in Latin America and the Caribbean: a systematic review. Brazilian Journal of Psychiatry, 38(1), 73-85. doi: 10.1590/1516-4446-2015-1652

Meier, A., Csiernik, R., Warner., \& Forchuk, C. (2015). The Stigma Scale: A Canadian Perspective. Social Work Research, 39(4), 213-222. doi: $10.1093 / \mathrm{swr} / \mathrm{svv} 028$

Mizuno, M., Yamaguchi, S., Taneda, A., Hori, H., A., Aikawa, A., \& Fujii, C. (2017). Development of Japanese version of King's Stigma Scale and its short version: Psychometric properties of a self-stigma measure. Psychiatry Clinical Neuroscience, 71(3), 189-197. doi: 10.1111/ pcn. 12470

Morandi, S., Gibellini-Manetti, S., Zimmermann, G., Favrod, J., Chanachev, A., Monnat, M., \& Bonsack, C. (2013). French translation, validation and adaptation of the Stigma Scale. Encephale, 39(6), 408-415. doi: 10.1016/j.encep.2013.03.002

Peluso, É. T. P., \& Blay, S. L. (2011). Public stigma and schizophrenia in São Paulo city. Brazilian Journal of Psychiatry, 33(2), 130-136. doi: 10.1590/S1516-44462010005000004

Picco, L., Lau, Y. W., Pang, S., Abdin, E., Vaingankar, J. A., Chong, S. A., \& Subramaniam, M. (2017). Mediating effects of self-stigma on the relationship between perceived stigma and psychosocial outcomes among psychiatric outpatients: Findings from a cross-sectional survey in Singapore. BMJ open, 7(8), 1-10. doi: 10.1136/bmjopen-2017-018228

R Core Team (2018). R: A language and environment for statistical computing. R Foundation for Statistical Computing, Vienna, Austria.

Regnerus, M. (2017). Is structural stigma's effect on the mortality of sexual minorities robust? A failure to replicate the results of a published study. Social Science \& Medicine, 188,157-165. doi: 10.1016/j.socscimed.2016.11.018

Reynoso, S. F., Dávalos, R. M., \& García, R. R. (2011). Estudio de traducción al español y evaluación psicométrica de una escala para medir el estigma internalizado en pacientes con trastornos mentales graves. Salud Mental, 34(4), 333-339. Recuperado de http://www.scielo. org.mx/pdf/sm/v34n4/v34n4a6.pdf

Sarkin, A., Lale, R., Sklar, M., Center, K. C., Gilmer, T., Fowler, C., ... Ojeda, V. D. (2015). Stigma experienced by people using mental health services in San Diego County. Social Psychiatry and Psychiatric Epidemiology, 50(5), 747-756. doi: 10.1007/s00127-014-0979-9

Teresi, J. A. (2001). Statistical methods for examination of differential item functioning (DIF) with applications to cross-cultural measurement of functional, physical and mental health. Journal of Mental Health and Aging, 7(1), 31-40. Recuperado de https://psycnet. apa.org/record/2001-06354-003

Urbina, S. (2007). Fundamentos da testagem psicológica. Porto Alegre: Artmed.

Wahl, O. F. (1999). Mental health consumers' experiences of stigma. Schizophrenia Bulletin, 25, 467-478. Recuperado de https://pdfs. semanticscholar.org/b5b0/a5e5e59ab1bc26892ea06d2d0560350b6254.pdf

Weber, C. A. T., \& Juruena, M. F. (2017). Paradigmas de atenção e estigma da doença mental na reforma psiquiátrica brasileira. Psicologia, Saúde e Doenças, 18(3), 640-656. doi: 10.15309/17psd180302

Zumbo, B. D., Gadermann, A. M., \& Zeisser, C. (2007). Ordinal versions of coefficients alpha and theta for Likert rating scales. Journal of modern applied statistical methods, 6(1), 4. doi: 10.22237/jmasm/117799218

Apêndice A

Figura de Suporte

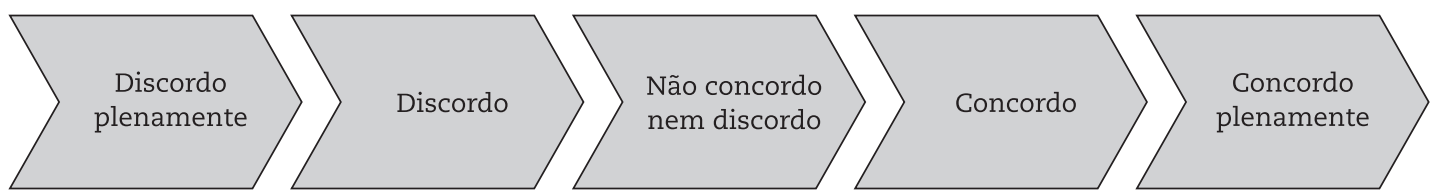

Apêndice B

Código utilizado para obtenção do índice de concordância entre os juízes por meio do Light's Kappa no Software R

\author{
library(irr) \\ kappam.light() \\ ?kappam.light \\ concjuizes<-read.csv(file.choose(),sep=";") \\ View(nicb) \\ kappa_juizes<-kappam.light(concjuizes) \\ kappa_juizes
}

Light's Kappa for m Raters 
Apêndice B (continuação)

Código utilizado para obtenção do índice de concordância entre os juízes por meio do Light’s Kappa no Software R

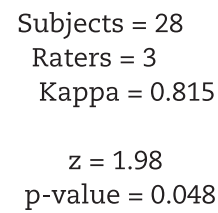

Apêndice C

Escala de Estigma da Doença Mental (EEDM)

Você encontrará abaixo uma lista de afirmações. Você deve assinalar, para cada uma delas, a resposta que melhor lhe descreve. Responda todas as questões sem exceção. Não gaste muito tempo pensando na resposta, a sua primeira impressão é a mais importante.

Questão

1. Eu fui discriminado durante os estudos devido aos meus problemas de saúde mental (A)

2. Às vezes sinto que estão falando de mim devido aos meus problemas de saúde mental (A)

3. Ter tido problemas de saúde mental me tornou uma pessoa mais compreensiva (D)

4. Eu não me sinto mal por ter tido problemas de saúde mental (D)

5. Tenho receio de dizer as pessoas que faço tratamento psicológico (A)

6. Algumas pessoas com problemas de saúde mental são perigosas (A)

7. As pessoas têm sido compreensivas com meus problemas de saúde mental (D)

8. Fui discriminado pela polícia por causa dos meus problemas de saúde mental (A)

9. Fui discriminado por meus chefes por causa dos meus problemas de saúde mental (A)

10. Meus problemas de saúde mental me fizeram aceitar melhor as outras pessoas (D)

11. Muitas vezes eu me sinto sozinho por causa dos meus problemas de saúde mental (A)

12. Tenho medo de como outras pessoas irão reagir se descobrirem sobre meus problemas de saúde mental (A)

13. Eu teria tido melhores chances na vida se não tivesse tido problemas de saúde mental (A)

14. Eu não me importo que as pessoas da minha vizinhança saibam que já tive problemas de saúde mental (D)

\begin{tabular}{cccc}
\multicolumn{5}{c}{ Resposta } & \\
\hline Concordo & Concordo & Não concordo \\
plenamente & nem discordo & Discordo & Discordo \\
plenamente
\end{tabular}

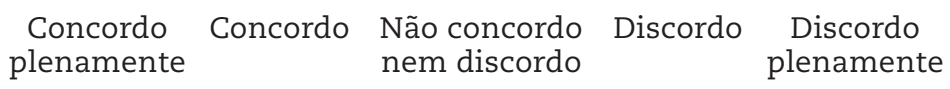

\begin{tabular}{|c|c|c|c|c|}
\hline $\begin{array}{l}\text { Concordo } \\
\text { plenamente }\end{array}$ & Concordo & $\begin{array}{l}\text { Não concordo } \\
\text { nem discordo }\end{array}$ & Discordo & $\begin{array}{c}\text { Discordo } \\
\text { plenamente }\end{array}$ \\
\hline $\begin{array}{l}\text { Concordo } \\
\text { plenamente }\end{array}$ & Concordo & $\begin{array}{l}\text { Não concordo } \\
\text { nem discordo }\end{array}$ & Discordo & $\begin{array}{c}\text { Discordo } \\
\text { plenamente }\end{array}$ \\
\hline $\begin{array}{l}\text { Concordo } \\
\text { plenamente }\end{array}$ & Concordo & $\begin{array}{l}\text { Não concordo } \\
\text { nem discordo }\end{array}$ & Discordo & $\begin{array}{c}\text { Discordo } \\
\text { plenamente }\end{array}$ \\
\hline $\begin{array}{l}\text { Concordo } \\
\text { plenamente }\end{array}$ & Concordo & $\begin{array}{l}\text { Não concordo } \\
\text { nem discordo }\end{array}$ & Discordo & $\begin{array}{c}\text { Discordo } \\
\text { plenamente }\end{array}$ \\
\hline $\begin{array}{l}\text { Concordo } \\
\text { plenamente }\end{array}$ & Concordo & $\begin{array}{l}\text { Não concordo } \\
\text { nem discordo }\end{array}$ & Discordo & $\begin{array}{c}\text { Discordo } \\
\text { plenamente }\end{array}$ \\
\hline $\begin{array}{l}\text { Concordo } \\
\text { plenamente }\end{array}$ & Concordo & $\begin{array}{l}\text { Não concordo } \\
\text { nem discordo }\end{array}$ & Discordo & $\begin{array}{c}\text { Discordo } \\
\text { plenamente }\end{array}$ \\
\hline $\begin{array}{l}\text { Concordo } \\
\text { plenament }\end{array}$ & Concordo & $\begin{array}{l}\text { Não concordo } \\
\text { nem discordo }\end{array}$ & Discordo & $\begin{array}{c}\text { Discordo } \\
\text { plenamente }\end{array}$ \\
\hline
\end{tabular}

Concordo Concordo Não concordo Discordo Discordo plenamente nem discordo plenamente

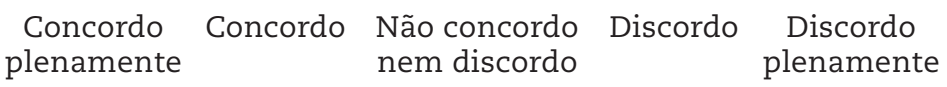

Concordo Concordo Não concordo Discordo Discordo plenamente nem discordo plenamente

$\begin{array}{crc}\begin{array}{c}\text { Concordo Concordo } \\ \text { plenamente }\end{array} & \begin{array}{l}\text { Não concordo Discordo } \\ \text { nem discordo }\end{array} & \begin{array}{c}\text { Discordo } \\ \text { plenamente }\end{array} \\ \begin{array}{c}\text { Concordo Concordo } \\ \text { plenamente }\end{array} & \begin{array}{l}\text { Não concordo Discordo } \\ \text { nem discordo }\end{array} & \begin{array}{c}\text { Discordo } \\ \text { plenamente }\end{array}\end{array}$


Apêndice C (continuação)

Escala de Estigma da Doença Mental (EEDM)

Você encontrará abaixo uma lista de afirmações. Você deve assinalar, para cada uma delas, a resposta que melhor lhe descreve. Responda todas as questões sem exceção. Não gaste muito tempo pensando na resposta, a sua primeira impressão é a mais importante.

\begin{tabular}{|c|c|c|c|c|c|}
\hline Questão & \multicolumn{5}{|c|}{ Resposta } \\
\hline $\begin{array}{l}\text { 15. Eu diria que tive problemas de saúde } \\
\text { mental se estivesse em uma entrevista } \\
\text { de emprego (D) }\end{array}$ & $\begin{array}{l}\text { Concordo } \\
\text { plenamente }\end{array}$ & Concordo & $\begin{array}{l}\text { Não concordo } \\
\text { nem discordo }\end{array}$ & Discordo & $\begin{array}{l}\text { Discordo } \\
\text { plenamente }\end{array}$ \\
\hline $\begin{array}{l}\text { 16. Tenho receio de dizer às pessoas que } \\
\text { tomo medicamentos para problemas de } \\
\text { saúde mental (A) }\end{array}$ & $\begin{array}{l}\text { Concordo } \\
\text { plenamente }\end{array}$ & Concordo & $\begin{array}{l}\text { Não concordo } \\
\text { nem discordo }\end{array}$ & Discordo & $\begin{array}{l}\text { Discordo } \\
\text { plenamente }\end{array}$ \\
\hline $\begin{array}{l}\text { 17. A reação das pessoas aos meus } \\
\text { problemas de saúde mental fazem com } \\
\text { que eu me isole }(\mathrm{A})\end{array}$ & $\begin{array}{l}\text { Concordo } \\
\text { plenamente }\end{array}$ & Concordo & $\begin{array}{l}\text { Não cc } \\
\text { nem d }\end{array}$ & Discordo & $\begin{array}{l}\text { Discordo } \\
\text { plenamente }\end{array}$ \\
\hline $\begin{array}{l}\text { 18. Eu sinto raiva da maneira que as } \\
\text { pessoas reagiram aos meus problemas } \\
\text { de saúde mental (A) }\end{array}$ & $\begin{array}{l}\text { Concordo } \\
\text { plenamente }\end{array}$ & rdo & $\begin{array}{l}\text { Não cc } \\
\text { nem d }\end{array}$ & Disc & $\begin{array}{l}\text { Discordo } \\
\text { plenamente }\end{array}$ \\
\hline $\begin{array}{l}\text { 19. Eu não tive nenhum problema com as } \\
\text { pessoas devido aos meus problemas de } \\
\text { saúde mental (D) }\end{array}$ & $\begin{array}{l}\text { Concordo } \\
\text { plenamente }\end{array}$ & Concordo & $\begin{array}{l}\text { Não } \\
\text { nem }\end{array}$ & Discordo & $\begin{array}{l}\text { Discordo } \\
\text { plenamente }\end{array}$ \\
\hline $\begin{array}{l}\text { 20. Já me senti discriminado por } \\
\text { profissionais de saúde devido aos meus } \\
\text { problemas de saúde mental (A) }\end{array}$ & $\begin{array}{l}\text { Concordo } \\
\text { plenamente }\end{array}$ & $\mathrm{Co}$ & $\begin{array}{l}\text { Não } \\
\text { nem }\end{array}$ & rdo & $\begin{array}{l}\text { Discordo } \\
\text { plenamente }\end{array}$ \\
\hline $\begin{array}{l}\text { 21. Já me evitaram por causa dos meus } \\
\text { problemas de saúde mental (A) }\end{array}$ & $\begin{array}{l}\text { Concordo } \\
\text { plenamente }\end{array}$ & Co & $\begin{array}{l}\text { Não concordo } \\
\text { nem discordo }\end{array}$ & rdo & $\begin{array}{l}\text { Discordo } \\
\text { plenamente }\end{array}$ \\
\hline $\begin{array}{l}\text { 22. Já me insultaram por causa dos meus } \\
\text { problemas de saúde mental (A) }\end{array}$ & $\begin{array}{l}\text { Concordo } \\
\text { plenamente }\end{array}$ & Concordo & $\begin{array}{l}\text { Não concordo } \\
\text { nem discordo }\end{array}$ & Discordo & $\begin{array}{l}\text { Discordo } \\
\text { plenamente }\end{array}$ \\
\hline $\begin{array}{l}\text { 23. Ter tido problemas de saúde mental me } \\
\text { fez uma pessoa mais forte (D) }\end{array}$ & $\begin{array}{l}\text { Concordo } \\
\text { plenamente }\end{array}$ & Concordo & $\begin{array}{l}\text { Não concordo } \\
\text { nem discordo }\end{array}$ & Discordo & $\begin{array}{l}\text { Discordo } \\
\text { plenamente }\end{array}$ \\
\hline $\begin{array}{l}\text { 24. Eu não me sinto constrangido por causa } \\
\text { dos meus problemas de saúde mental (D) }\end{array}$ & $\begin{array}{l}\text { Concordo } \\
\text { plenamente }\end{array}$ & Concordo & $\begin{array}{l}\text { Não concordo } \\
\text { nem discordo }\end{array}$ & Discordo & $\begin{array}{l}\text { Discordo } \\
\text { plenamente }\end{array}$ \\
\hline $\begin{array}{l}\text { 25. Eu evito contar às pessoas sobre os } \\
\text { meus problemas de saúde mental (A) }\end{array}$ & $\begin{array}{l}\text { Concordo } \\
\text { plenamente }\end{array}$ & Concordo & $\begin{array}{l}\text { Não concordo } \\
\text { nem discordo }\end{array}$ & Discordo & $\begin{array}{l}\text { Discordo } \\
\text { plenamente }\end{array}$ \\
\hline $\begin{array}{l}\text { 26. Ter tido problemas de saúde mental me } \\
\text { faz sentir que a vida é injusta (A) }\end{array}$ & $\begin{array}{l}\text { Concordo } \\
\text { plenamente }\end{array}$ & Concordo & $\begin{array}{l}\text { Não concordo } \\
\text { nem discordo }\end{array}$ & Discordo & $\begin{array}{l}\text { Discordo } \\
\text { plenamente }\end{array}$ \\
\hline $\begin{array}{l}\text { 27. Eu sinto a necessidade de esconder } \\
\text { meus problemas de saúde mental dos } \\
\text { meus amigos (A) }\end{array}$ & $\begin{array}{l}\text { Concordo } \\
\text { plenamente }\end{array}$ & Concordo & $\begin{array}{l}\text { Não concordo } \\
\text { nem discordo }\end{array}$ & Discordo & $\begin{array}{c}\text { Discordo } \\
\text { plenamente }\end{array}$ \\
\hline $\begin{array}{l}\text { 28. Eu acho difícil dizer às pessoas que } \\
\text { tenho problemas de saúde mental (A) }\end{array}$ & $\begin{array}{l}\text { Concordo } \\
\text { plenamente }\end{array}$ & Concordo & $\begin{array}{l}\text { Não concordo } \\
\text { nem discordo }\end{array}$ & Discordo & $\begin{array}{l}\text { Discordo } \\
\text { plenamente }\end{array}$ \\
\hline
\end{tabular}

Apêndice D

Código utilizado para obtenção da consistência interna da EEDM (Alpha Ordinal) no Software R

eedm<-read.csv(file.choose(),sep=";")

View (eedm)

library(psych)

alpha(cor_auto(ss[,c(1,2,8,9,11,13,17,18,19,20,21,22,26)]),n.obs=23,check.keys=TRUE)

\#RCall: alpha(x = cor_auto(ss[, c(1, 2, 8, 9, 11, 13, 17, 18, 19, 20,

\# $\quad 21,22,26)])$, check.keys = TRUE, n.obs = 23)

\#raw_alpha std.alpha G6(smc) average_r S/N ase 
Apêndice D (continuação)

Código utilizado para obtenção da consistência interna da EEDM (Alpha Ordinal) no Software R

$\begin{array}{lllll}\# 0.8 & 0.8 & 1 & 0.23 & 40.062\end{array}$

\#lower alpha upper $95 \%$ confidence boundaries

\#0.68 0.80 .92

alpha(cor_auto(ss[,c(4,5,12,14,15,16,24,25,27,28)]),n.obs=23,check.keys=TRUE)

\#Call: alpha(x = cor_auto(ss[, c(4, 5, 12, 14, 15, 16, 24, 25, 27, 28)]),

\# $\quad$ check.keys = TRUE, n.obs = 23)

\#raw_alpha std.alpha G6(smc) average_r $\mathrm{S} / \mathrm{N}$ ase

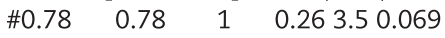

\#lower alpha upper $95 \%$ confidence boundaries

\#0.64 0.780 .91

alpha(cor_auto(ss[,c(3,6,7,10,23)]),n.obs=23,check.keys=TRUE)

\#Call: alpha(x = cor_auto(ss[, c $(3,6,7,10,23)])$, check.keys = TRUE,

\# n.obs $=23$ )

\#raw_alpha std.alpha G6(smc) average_r S/N ase

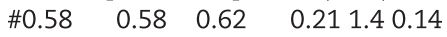

\#lower alpha upper $95 \%$ confidence boundaries

\#0.3 0.580 .85

alpha(cor_auto(ss),n.obs=23, check.keys $=$ TRUE)

\#Call: alpha $(\mathrm{x}=$ cor_auto(ss), check.keys $=$ TRUE, n.obs $=23$ )

\#raw_alpha std.alpha G6(smc) average_r S/N ase

$\begin{array}{llll}\# 0.87 & 0.87 & 1 & 0.19 \\ & & 6.6 & 0.04\end{array}$

\#lower alpha upper $95 \%$ confidence boundaries

$\# 0.790 .870 .95$

\section{Sobre os autores}

Nicolas de Oliveira Cardoso é psicólogo (ULBRA), Mestre em psicologia clínica pela Pontifícia Universidade Católica do Rio Grande do Sul (PUCRS). Atualmente é doutorando em psicologia clínica pela PUCRS.

Breno Sanvicente-Vieira é psicólogo (PUCRS), mestre e doutor em psicologia (PUCRS). Atualmente é professor associado do Departamento de Psicologia da Pontifícia Universidade Católica do Rio de Janeiro (PUC-Rio).

Wagner de Lara Machado é psicólogo (ULBRA), doutor em psicologia (UFRGS), Pós-doutor como consultor de análise quantitativa de dados e psicometria (UFRGS). Atualmente é professor nos cursos de Graduação e Pós-Graduação em Psicologia da PUCRS.

Irani Iracema de Lima Argimon é psicóloga (PUCRS), doutora em psicologia (PUCRS). Atualmente é professora e coordenadora do programa de Pós-Graduação em Psicologia da PUCRS.

\section{Como citar este artigo}

Cardoso et al. (2020). Adaptação Transcultural da The Stigma Scale para o Português Brasileiro: Estudo Piloto. Avaliação Psicológica, 19(3), 254-267. http://dx.doi.org/10.15689/ap.2020.1903.17467.04 